VI. GENERATION OF SOLAR MAGNETIC FIELDS 


\title{
ORDER AND CHAOS IN THE SOLAR CYCLE
}

\author{
A.A. RUZMAIKIN \\ Institute of Terrestrial Magnetism, \\ Ionosphere and Radio Wave Propagation \\ Academy of Sciences USSR \\ 142092, Troitsk, Moscow Region, USSR
}

\begin{abstract}
Solar activity varying with an 11-year cycle is chaotic at large time scales. The evidence comes from an analysis of observations of the sunspot number and of radioactive carbon. Thereby an estimate of the dimension of the solar attractor can be obtained.

The origin of the sunspots can be associated with the interactions of the regular, large-scale, chaotic, and intermittent magnetic fields.
\end{abstract}

\section{Introduction}

Before the nineteenth century solar activity was observed by people as random events, mainly through the appearance of sunspots. H. Schwabe was the first who discovered the 11-year cyclicity in the middle of the 19th century. Since that time solar scientists have been looking for the order in the solar activity.

Until the present time the standard point of view has been the following: There is order in the large scales of space and time, e.g. larger than a tenth of the solar radius and one year. For example an annual average of the Maunder butterfly diagram for the sunspot latitude distribution represents order. Then there is disorder in the small scales, e.g. a single spot appears and disappears randomly.

Modern knowledge has cast new light on this issue. It appears that the activity becomes chaotic at large time scales, say, hundreds of years. For example, there were no sunspots during the epochs of the Grand Minima, which occur randomly in time. The concept of global stochasticity of solar activity was introduced eight years ago (Ruzmaikin, 1981; see also Zeldovich and Ruzmaikin, 1983). The origin of chaotic behaviour can be associated with a strange attractor in the phase space of the solar dynamical system.

At the same time there exist some quasi-ordered structures at small scales, for example the network of granulation and mesogranulation.

In that sense the active Sun is a kingdom of physics. Its picture resembles the Arabian makama, a genre of literature that mixes prose and verse, erudition and knavish trickery, sermon and joke. Here I will try to demonstrate this picture by crude strokes with the MHD brush. It is magnetohydrodynamic because convection of the conducting fluid and the magnetic field is responsible for all active phenomena on the Sun. 


\section{Solar Attractor}

Let us first consider the processes averaged over a time scale exceeding a year and a spatial scale exceeding the basic scale of solar convection, the diameter of a supergranule. The motions and magnetic fields evolving in the convection zone represent an averaged solar dynamical system. The phase space of the dynamical system can be described, e.g. by the components of the mean (large-scale) magnetic field and the mean velocity field. A path in this phase space corresponds to some solution of the averaged MHD equations. Fortunately it is not necessary to investigate all paths (solutions), because normally there are some manifolds in phase space to which all or almost all paths attract. It is sufficient to find the attractors in order to know the behaviour of the dynamical system. This problem is also very difficult. However, some important characteristics of the attractor can easily be found directly from the observational data. The dimension of the attractor can for instance be determined.

\subsection{DIMENSION}

The simplest attractors have an integer dimension. The attractor of dimension $d=0$ corresponds to a point. If such an attractor were present at the origin of the dynamical system under consideration, the Sun would not have any large-scale magnetic field and motions. A closed curve as an attractor of dimension $d=1$ is the limiting Poincaré cycle. It is exactly the 11-year cycle if it can be stable in time. The attractor of dimension $d=2$, a so-called 2 -torus, may describe the 11-year cycle as modulated by a secular variation. The trajectories of the paths are regular for attractors of integer dimensions.

More typical are however attractors of fractal dimensions. Crudely one can imagine an attractor of fractal dimension, e.g. $d=3-\epsilon$, where $\epsilon \ll 1$, as a ball with an infinite number of small holes in it. A path travelling across this manifold will certainly seem very irregular or chaotic. A well known example is the Lorenz attractor (Lorenz, 1963), which has $d=2.09$ (something more than a surface). The dimension of the attractor is a measure of the regular or chaotic behaviour of the dynamical system.

Is it possible to find the dimension from observational data? At a first glance it appears impossible when one as usual only knows the time behaviour of a single quantity (a projection in phase space). One may, however, hope that a sequence of values sufficiently prolonged in time will reveal the basic properties of the attractor. Grassberger and Procaccia (1983) suggested a method of finding the attractor dimension by using a time series of the observed quantity $x(t), x(t+\tau), \ldots, x[t+(N-1) \tau]$, with a given time increment $\tau$. The attractor sought for must be embedded in a phase space of a large dimension $m$, defined by the vectors

$$
\xi_{i}(t)=[x(t), x(t+\tau), \ldots, x(t+(m-1) \tau)], \quad i=1,2, \ldots, N-1 .
$$

The dimension $d$ of the attractor is defined as the exponent of the correlation integral $w(r) \sim$ $r^{d}$ for small distances $r$ between the points $\xi_{i}$.

$$
w(r)=N^{-2} \sum_{i, j=1}^{N} \theta\left(r-\left|\xi_{i}-\xi_{j}\right|\right),
$$

where $N$ is the total number of experimental points used, and $\theta$ is the Heaviside function. Thus, to find the dimension one needs to isolate for small $r$ a linear part of the curve $(\log w$ 
vs. $\log r)$, and to determine its inclination.

Applying this method to the series of relative sunspot numbers, a dimension close to 2 has been obtained (Kürths, 1987; Makarenko and Ajmanova, 1989). The series of sunspot numbers is, however, too short to include such features as the Grand Minima. It is better to use more extended sequences, such as the carbon isotope ${ }^{14} C$ abundance in the annual tree rings available over 9000 years (Suess, 1965, 1978; Damon and Sonett, 1989; Dergachev, private communication). The rate of ${ }^{14} C$ formation in the Earth's atmosphere due to the bombardment by cosmic ray particles is well anti-correlated with the number of sunspots, as a result of the solar magnetic field acting as a filter for the $\operatorname{cosmic}$ rays. Thus ${ }^{14} \mathrm{C}$ is an index of solar activity.

An estimate of the attractor dimension from Suess's data over 6000 years gives $d \approx 3.3$ (Gizzatulina et al., 1988), i.e., the dimension is close to 3. Because the 3 -torus according to the theory of stochastic dynamical systems is unstable and will be transformed into a strange attractor, the above result means that the mean solar attractor is fractal, i.e., the dynamical system is chaotic. The estimate of the dimension can be improved by using more extended and accurate data. The first estimates of the attractor dimension indicate a low dimension. This also suggests that the mean solar dynamical system is very simple. It can be described in terms of only few effective modes. Let us consider the probable origin of these modes (Ruzmaikin, 1985).

\subsection{2-YEAR SOLAR CYCLE AS A MANIFESTATION OF DYNAMO WAVES}

One of the possible effective modes of the mean solar dynamical system is the 22-year magnetic cycle. The analysis of the annual mean values of the Wolf sunspot numbers $W$ for 19 of the 11-year cycles made by Gudzenko and Chertoprud (1964) shows the existence of a limiting cycle of near elliptical form in phase space $(W, \mathrm{~d} W / \mathrm{d} t)$. This analysis assumes the existence of the derivative $\mathrm{d} W / \mathrm{d} t$ and hence excludes the possibility for the attractor to be fractal (Makarenko and Ajmanova, 1989). However, as a smooth approximation over a finite time interval it looks reasonable and gives the possibility of extracting one of the effective modes of the dynamical system.

This two-parameter limiting cycle can be explained by the mean field dynamo theory (cf. Krause and Rädler, 1980). The mean magnetic field is excited and evolves in the solar convective zone due to the differential rotation $(\nabla \Omega)$, mean helicity $(\alpha)$, and turbulent diffusion $\left(\nu_{T}\right)$ :

$$
\begin{aligned}
\frac{\partial A}{\partial t} & =\alpha B_{\phi}+\nu_{T} \nabla^{2} A \\
\frac{\partial B_{\phi}}{\partial t} & =r \sin \theta(\nabla \Omega \times \nabla A)_{\phi}+\nu_{T} \nabla^{2} B_{\phi}
\end{aligned}
$$

where $A$ is the azimuth component of the vector potential of the poloidal field $\left(B_{r}, B_{\theta}\right)$.

The solution has the form of waves propagating, when the value of $\nu_{T} /(\alpha R)$ is small, along the surfaces of constant angular velocity (Yoshimura, 1975). For instance, when the angular velocity only depends on the radial coordinate, the dynamo wave travels along the solar surface:

$$
A=A(\omega t-k \theta), \quad B_{\phi}=B_{\phi}(\omega t-k \theta+\delta) .
$$


The direction of propagation is determined by the sign of the dimensionless dynamo number $D=R_{\odot}^{3}(\sin \theta) \alpha_{\max }(\mathrm{d} \Omega / \mathrm{d} r)_{\max } / \nu_{T}^{2}$. The wave propagates from the pole to the equator when $D$ is negative, and to the pole when $D$ is positive. The frequency of the wave is $\omega=(k|D| / 2)^{1 / 2}$, the phase speed is $\omega / k=(|D| / 2 k)^{1 / 2}$, and the group speed is a factor of two smaller than the phase speed. The phase shift between the poloidal and toroidal components of the magnetic field is $\delta$, which is close to $\pi / 4$ in the standard kinematic models of the solar dynamo (cf. Parker, 1979; Krause and Rädler, 1980). In this case the radial component of the field is lagging behind $B_{\phi}$ by $3 \pi / 4$.

In the other idealized case when $\Omega$ is only dependent on $\theta$, the dynamo wave propagates radially. It is of importance to know the real distribution of the angular velocity as well as the mean helicity in the solar convection zone. According to mixing length models of stellar convection zones (Spruit, 1974), the turbulent diffusivity can be considered as almost constant in the bulk of the solar convection zone.

Makarov et al. (1987) (cf. also Brandenburg and Tuominen, 1988), have made an attempt to find the dynamo wave distribution on the solar surface by using the depth dependence of the angular velocity obtained from current helioseismology data (Fig.1) and the mean helicity estimated via the mixing-length theory. They found that the source for generating the mean magnetic field, $\alpha r(\mathrm{~d} \Omega / \mathrm{d} r)$, has three main maxima in the convection zone: near the solar surface at $0.9 R_{\odot}$, at $0.8 R_{\odot}$, and close to the bottom at $0.7 R_{\odot}$.

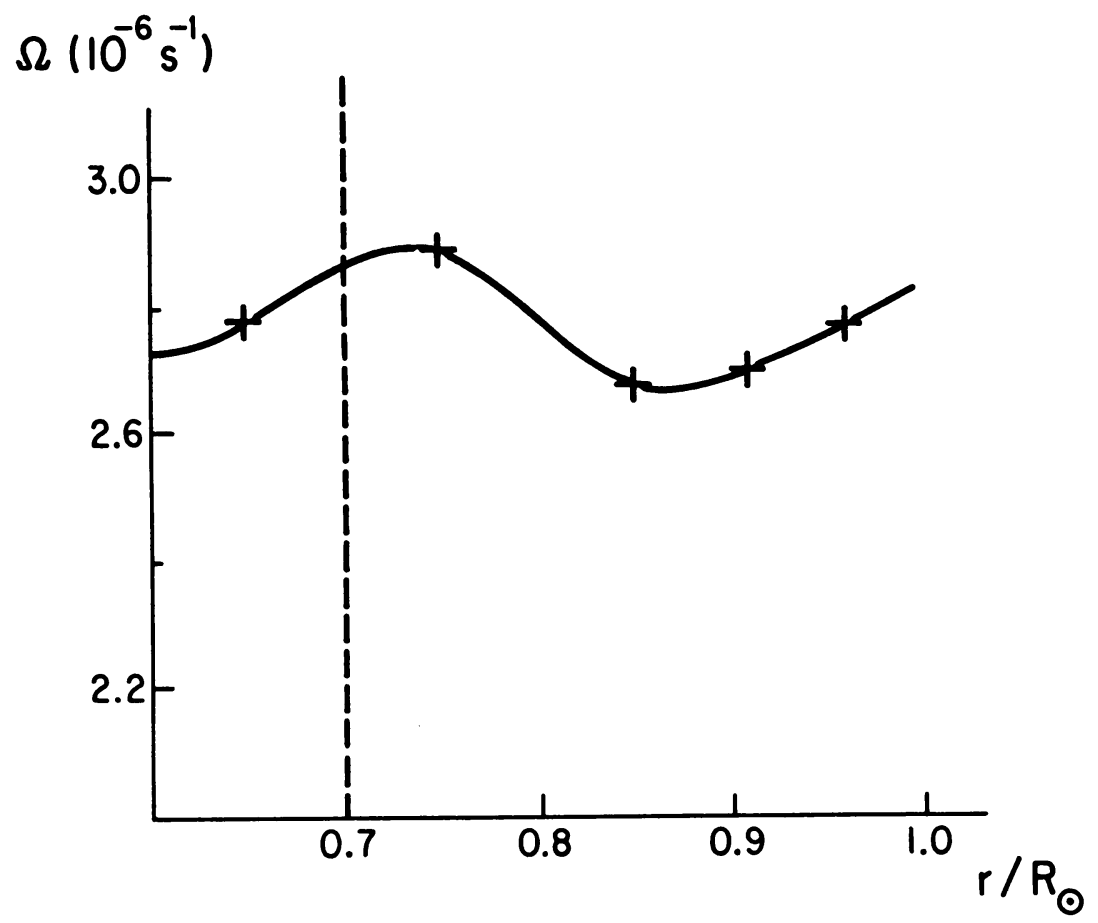

Figure 1. The radial profile of the angular velocity in the solar convection zone appears to have regions with different sign of the gradient. 


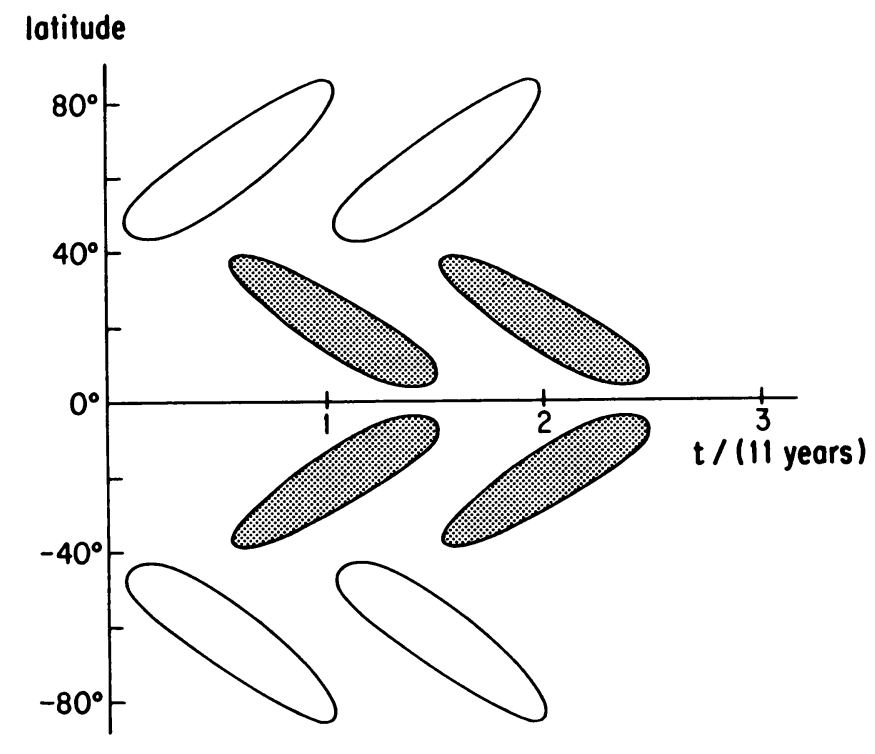

Figure 2. Schematic representation of two magnetic waves on the solar surface (the polar and equatorial dynamo waves, produced by the action of mean helicity and differential rotation of the type given in Fig.1).

In the first region the value of $\alpha(\mathrm{d} \Omega / \mathrm{d} r)$ is positive, so that the dynamo wave originating there propagates towards the pole in each hemisphere. The maximum of the wave amplitude is reached approximately at a latitude of $65^{\circ}$. This wave probably manifests itself in the activity of polar faculae migrating to the pole. The second maximum, at $0.8 \mathrm{R}_{\odot}$, has $\alpha(d \Omega / d r)<0$. It is larger and creates a wave that is ten times larger in amplitude, and which migrates to the equator. This wave is naturally associated with the standard Maunder butterfly diagram of sunspot activity (Fig. 2). The third and deepest maximum of the source also creates a wave propagating towards the pole. This wave is, however, rather weak on the solar surface due to the considerable depth of the source. Makarov et al. (1987) have associated this wave with the apparent extra reversals of the poloidal magnetic field. Let us finally mention an important element of order, which was pointed out by Stenflo (1988). The observed odd rotationally symmetric modes of the solar magnetic field vary with the $22 \mathrm{yr}$ period, while this period is absent for the even modes. This fact agrees with the symmetry and preferable excitation of odd modes in solar dynamo theory.

\subsection{ROTATIONAL WAVES AND MERIDIONAL FLOWS}

Observational evidence have been found for zones of higher and lower rotational velocities (3-6 $\mathrm{m} \mathrm{s}^{-1}$ in amplitude), propagating with an 11-year period, superposed on the general differential rotation (LaBonte and Howard, 1982). The study of sunspot motions have shown meridional flows with the same periodicity (Tuominen et al., 1983). 
The strong correlation between the motions and the large-scale magnetic field suggests that the motions are created due to the action of dynamo waves. A simple picture of a rotational wave produced by a magnetic dynamo wave has been drawn by Kleeorin and Ruzmaikin (1984 a) as follows:

The rotational velocity variations produced by the magnetic field can be estimated from the balance between the turbulent and magnetic stresses,

$$
\rho \nu_{T} \frac{\partial}{\partial r}\left(\frac{u_{\phi}}{r}\right)=-\frac{B_{r} B_{\phi}}{4 \pi} .
$$

The magnetic field can be considered as given, because the rotational variations observed are small. In particular, in the case that $\Omega=\Omega(r)$, the field can be written in the form

$$
\begin{aligned}
& B_{r}=b_{r}(r, \theta) \cos (\omega t-k \theta-\pi / 2-\delta), \\
& B_{\phi}=b_{\phi}(r, \theta) \cos (\omega t-k \theta),
\end{aligned}
$$

where the amplitude distribution, frequency, and phase are found by solving the dynamo equations.

For the time-varying part of the rotational velocity one immediately obtains a wave with the double frequency,

$$
u_{\phi}=u_{0}(r, \theta) \cos (2 \omega t-2 k \theta+\pi / 2-\delta) .
$$

The distribution of the wave amplitude, $u_{0} \sim b_{r} b_{\phi}$, proves to be more homogeneous as compared with the distribution of the amplitude of the magnetic field. Let the azimuth component of the magnetic field (manifested on the surface in the form of sunspots) be concentrated to mid-latitudes, e.g. as $b_{\phi} \sim \sin 2 \theta$, on the solar surface, and let the radial component increase towards the poles, e.g. as $b_{r} \sim \sin \theta$. Then $u_{0} \sim \sin \theta \cos ^{2} \theta$ is distributed more homogeneously over the latitudes than the field. The phase of the maximum of the rotational wave is shifted as compared with the phase of the maximum magnetic flux (defined by $\left.\left|B_{\phi}\right|\right)$. The shift is $1 / 8$ of the wavelength of the rotational wave if $\delta=\pi / 4$, as the kinematic dynamo theory predicts. This consequence of our simple model is in good agreement with the observations of Howard and LaBonte.

For more details concerning the magnetic action on the rotation and meridional motion, we refer to the papers by Rüdiger et al. (1986) and Kleeorin and Ruzmaikin (1989).

\subsection{CAUSES FOR THE STOCHASTIC BEHAVIOUR OF SOLAR ACTIVITY}

The second effective mode of the mean solar dynamical system can be identified with the secular modulation of the 22-year solar cycle. Nonlinear beats between odd (dipole type) and even (quadrupole type) magnetic fields may provide a mechanism for the modulation (Kleeorin and Ruzmaikin, $1984 \mathrm{~b}$ ). Magnetic fields of both symmetries in the form of waves with closely spaced frequencies may be excited by dynamo action. The beats strongly compete with the synchronization that tends to equalize the frequencies. Perhaps this causes the observed irregularity of the secular modulation.

Another reason for this irregular variation is a possible decay of the magnetic field to zero. Actually the existence of a non-magnetic sun does not contradict the equilibrium conditions or other fundamentals. It may even be profitable from some point of view. However, the non-magnetic state is unstable due to the dynamo number (the amplitude of the source for the generation of magnetic fields in the solar convection zone) being sufficiently large. 
The magnetic field grows when the dynamo number is large. However, the growing field smoothes out the angular velocity gradients and removes the predominance of left-handed vortices over the right-handed ones, i.e., it reduces the mean helicity. As a result the effective dynamo number decreases and the dynamical system can fall down into a non-magnetic state. Some simple models demonstrating such a behaviour have been constructed (Ruzmaikin, 1981; Weiss et al., 1984; Malinetsky et al., 1986). In the first paper based on a Lorenz type model an estimate of the characteristic time to stay in the vicinity of the non-magnetic state is given by $\tau\left(D-D_{c r}\right)^{-1 / 2}$, where $\tau$ is the decay time of the mean helicity, and $D_{c r}$ is the value of the dynamo number at which excitation of dynamo waves starts. In the more developed model by Malinetsky et al. (1986) it is shown how groups of regular 22-years oscillations are separated by deep minima (Fig.3). The behaviour of the system in phase space is similar to the dynamics of the 3 -torus.

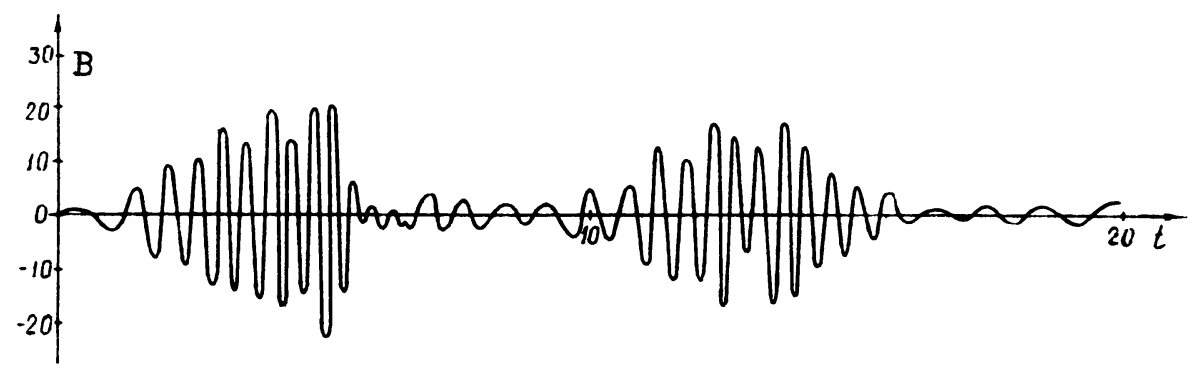

Figure 3. Time evolution of the mean magnetic field in the model of Malinetsky et al. (1986).

\section{Intermittency of solar magnetic fields}

Together with the mean magnetic field a fluctuating, small-scale magnetic field is excited. Observations provide evidence that the field is concentrated in thin magnetic flux tubes. The fluctuating field can also be associated with the origin of the sunspots. Let us consider briefly these two problems.

\subsection{DISTRIBUTION OF THE FLUCTUATING MAGNETIC FIELD}

Let $h_{i}(r, t)$ represent the deviation of the magnetic field from its mean value, and

$$
w(r)=<h_{i}\left(\mathbf{r}_{1}\right) h_{i}\left(\mathbf{r}_{2}\right)>, \quad r=\left|\mathbf{r}_{1}-\mathbf{r}_{2}\right|
$$

be a space correlation function of the field.

As was shown by Kleeorin et al. (1986) in the framework of a kinematic dynamo model with a velocity field having a short correlation scale, assuming a magnetic Reynolds number of the order of $10^{8}$ typical for the solar convection zone, two modes of the fluctuating magnetic field can be excited (Fig.4).

The first mode corresponds to a simple loop having a diameter comparable to the correlation scale of the turbulent convection $l \sim 10^{4} \mathrm{~km}$ (the size of a supergranule), and a 

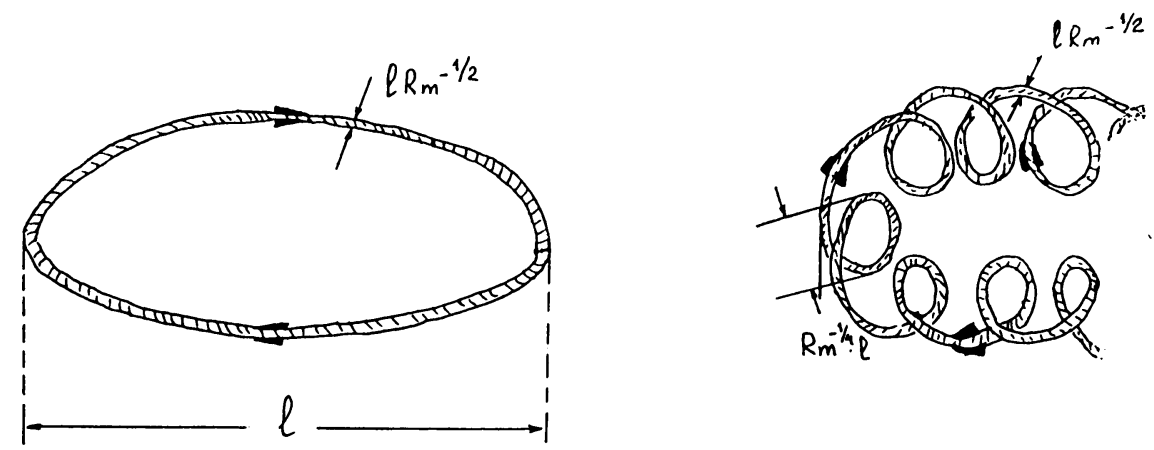

Figure 4. The modes of the fluctuating magnetic field that can be excited by dynamo action in the solar convection zone.

thickness of $l R_{m}^{-1 / 2} \sim 1 \mathrm{~km}$. The second mode is associated with a more complicated loop having a subset of smaller loops of diameter $l R_{m}^{-1 / 4} \sim 100 \mathrm{~km}$. This scale may be compared with the dimensions of observed flux tubes (Muller, 1985).

It is further necessary to take into account the cell structure of the solar convection. It causes advection and concentration of the magnetic field to the cell boundaries (Galloway et al., 1977).

The distribution of the magnetic field in a turbulent flow with a large magnetic Reynolds number should be very inhomogeneous from a fundamental point of view (Molchanov et al., 1985). The magnetic field in such a flow is concentrated into intermittent bundles with large regions in between with a relatively weak field. Thus the appearance of solar magnetic flux tubes can be considered as a natural manifestation of such a behaviour of the magnetic field due to the action of random motions of the highly conducting fluid.

\subsection{ON THE ORIGIN OF SUNSPOTS}

A sunspot is a region of reduced temperature (by at most $1500 \mathrm{~K}$ ). It originates randomly in the vicinity of the maximum of the large-scale magnetic field that evolves with the solar cycle. The total area covered by sunspots over the 11-year cycle does not exceed $2 \%$ of the solar surface.

Sunspots thus appear as a set of temperature spots. However, they are not like temperature spots in a turbulent flow, where excesses and deficits of the temperature are equally abundant.

The origin of sunspots should be associated with the magnetic field. We recall that solar magnetic fields were discovered by G. Hale in sunspots. A pair of sunspots is normally connected by a loop of the azimuthal magnetic field emerging through the solar surface, probably due to buoyancy (Parker, 1979). It is, however, not clear why separate loops and not the whole latitude belt of the azimuthal field emerge. Let us note that the magnetic field is rather irregular even inside sunspots, although the same field direction is predominant inside the spots (Bray and Loughead, 1969).

It is natural to associate the origin of sunspots with the intermittency of the solar magnetic field, i.e., with random, infrequent but intense concentrations of the fluctuating magnetic 
field generated by random motions. Deviations from the mean magnetic field occur both to increase and to decrease that field. However, only after an increase the total magnetic field (mean field plus fluctuation) can reach the critical value that makes it float due to buoyancy (Fig.5).

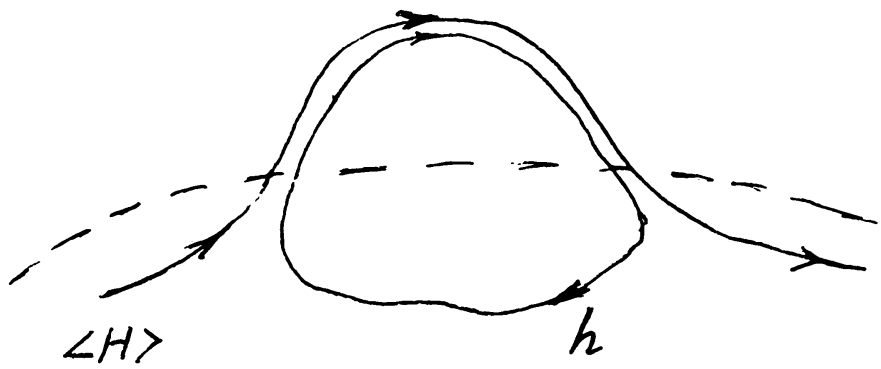

Figure 5. The sum of the mean $\langle H\rangle$ and the intermittent, fluctuating field $h$ may reach a critical value to allow the field to emerge from the solar convection zone.

The distribution of the intermittent magnetic field is close to a log-normal distribution (Zeldovich et al., 1987):

$$
P(\ln H)=\frac{1}{\sqrt{2 \pi} \sigma} \exp \left[\frac{-(\ln H-a)^{2}}{2 \sigma^{2}}\right],
$$

where $a=<\ln H>$ and $\sigma^{2}=<(\ln H-a)^{2}>$. The symbol $<>$ means 'mean value'. The successive statistical moments grow with increasing order $p$ :

$$
<H>=\exp \left(a+\sigma^{2} / 2\right),<H^{2}>=\exp \left(2 a+2 \sigma^{2}\right), \ldots,<H^{p}>=\exp \left(p a+p^{2} \sigma^{2}\right) .
$$

To eliminate the unknown value of $a$ it is better to use distribution for the normalized magnetic field $H_{n}=H /<H>$,

$$
P\left(H_{n}\right)=P(\ln H) \frac{\mathrm{d} \ln H}{\mathrm{~d} H_{n}}=\frac{1}{\sqrt{2 \pi} \sigma H_{n}} \exp \left[\frac{-\left(\ln H_{n}+\sigma^{2} / 2\right)^{2}}{2 \sigma^{2}}\right],
$$

determined by the single parameter $\sigma$.

The probability for the normalized field to exceed a critical value $\zeta$ is

$$
\begin{aligned}
& \operatorname{Prob}\left(H_{n}>\zeta\right)=\int_{\zeta}^{\infty} \frac{1}{\sqrt{2 \pi} \sigma H_{n}} \exp \left[\frac{-\left(\ln H_{n}+\sigma^{2} / 2\right)^{2}}{2 \sigma^{2}}\right] \mathrm{d} H_{n}= \\
& =\int_{\ln \zeta}^{\infty}(\sqrt{2 \pi} \sigma x)^{-1} \exp \left[\frac{-\left(x^{2}+\sigma^{2} / 2\right)}{2 \sigma^{2}}\right] \mathrm{d} x=(1 / 2)\left[1-F\left(\frac{\ln \zeta+\sigma^{2} / 2}{\sigma}\right)\right] .
\end{aligned}
$$


Equating this probability with the relative area covered by sunspots ( $1 \%$ of the total area of the Sun) one can obtain an estimate for the parameter $\sigma$. In principle this parameter should be determined from theory.

\section{References}

Brandenburg, A. and Tuominen, I. (1988) 'Variation of magnetic field and flows during the solar cycle', COSPAR paper No. 12.4.6, Espoo, Finland.

Bray, R.J. and Loughead, R.E. (1964), Sunspots, Chapman and Hall Ltd., London.

Damon, P. and Sonett, C.P. (1989) 'The spectrum of radiocarbon', in Proc. Int. Conf. 'Sun in Time', Arizona Univ. Press, Tucson.

Eddy, J.A. (1976) 'The Maunder Minimum', Science 192, 1189-1202.

Galloway, D.J., Proctor, M.R.E., and Weiss, N.O. (1977) 'Formation of the intense magnetic fields near the surface of the Sun', Nature 266, 686-692.

Gizzatulina, S.M., Ruzmaikin, A.A., Rukavishnikov, V.D., and Tavastsherna, K.S. (1988) 'Radiocarbon evidence of global stochasticity of solar activity', Preprint 40 (794), IZMIRAN, Moscow (submitted to Solar Phys.).

Grassberger, P. and Procaccia, I. (1983) 'Measuring the strangeness of strange attractors', Physica D9. 189-208.

Gudzenko, L.L. and Chertoprud, V.E. (1964) 'Some dynamical properties of solar activity', Soviet Astron. 41, 697-706.

Kleeorin, N.I. and Ruzmaikin, A.A. (1984 a) 'On the nature of 11-year torsional oscillations of the Sun', Pisma Astron.Zh. (USSR) 10, 925-935.

Kleeorin, N.I. and Ruzmaikin, A.A. (1984 b) 'Mean-field dynamo with cubic non-linearity', Astron. Nachr. 305, 265-275.

Kleeorin, N.I. and Ruzmaikin, A.A. (1989) 'Large-scale flows excited by magnetic fields in the solar convective zone', Preprint, IZMIRAN, Moscow.

Kleeorin, N.I., Ruzmaikin, A.A., and Sokoloff, D.D. (1986) 'Correlation properties of selfexciting fluctuating magnetic fields', Proc.Varenna-Abastumani Int.School 'Plasma Astrophysics', ESA SP-251-ISSN 0379-6566; (1988) Kinematics and Physics of Celestial Bodies 4, 28-35.

Krause, F. and Rädler, K.-H. (1980) Mean-field magnetohydrodynamics and dynamo theory, Akademie-Verlag, Berlin.

Kürths, J. (1987) 'Estimating parameters of attractors in some astrophysical time series', in M. Forkas (ed.), Proc. Int. Conf. on Nonlinear Oscillations, Budapest, pp.664-667.

LaBonte, B.J. and Howard, R. (1982) 'Torsional waves on the sun and the activity cycle', Solar Phys. 75, 161-178.

Lorenz, E.N. (1963) 'Deterministic nonperiodic flow', J. Atmosph. Sci. 20, 130-141.

Makarenko, N.G. and Ajmanova, G.K. (1989) 'K entropy and dimension of solar attractor', submitted to Pisma Astron. Zh.

Makarov, V.I., Ruzmaikin, A.A., and Starchenko, S.V. (1987) 'Magnetic waves of solar activity', Solar Phys. 111, 267-277.

Malinetsky, G.G., Ruzmaikin, A.A., and Samarsky, A.A. (1986) 'A model of longperiodic variations of solar activity', Preprint No. 170, Keldysh Inst. of Appl. Math., Moscow.

Molchanov, S.A., Ruzmaikin, A.A., and Sokoloff, D.D. (1985) 'Kinematic dynamo in random flow', Sov. Phys. Uspekhy 145, 593-628. 
Morfill, G. and Voges, W. (1989) 'The solar cycle: Deterministic or chaotic', in Proc. Int. Conf. 'Sun in Time', Arizona Univ. Press, Tucson.

Muller, R. (1985) 'The fine structure of the quiet Sun', Solar Phys. 100, 237-255.

Parker, E.N. (1979) Cosmic Magnetic Fields, Clarendon Press, Oxford.

Rüdiger, G., Tuominen, I., Krause, F., and Virtanen, H. (1986) 'Dynamo-generated flows in the Sun: 1. Foundation and first results', Astron. Astrophys. 166, 306-318.

Ruzmaikin, A.A. (1981) 'Solar cycle as strange attractor', Comments on Astrophys. 9, 85-93.

Ruzmaikin, A.A. (1985) 'The solar dynamo', Solar Phys. 100, 125-140.

Spruit, H.C. (1974) 'A model of the solar convection zone', Solar Phys. 34, 277-290.

Stenflo, J.O. (1988) 'Global wave patterns in the Sun's magnetic field', Astrophys. Space Sci. 144, 321-336.

Suess, H.E. (1965) 'Secular variations of cosmic-ray produced carbon 14 in the atmosphere and their interpretations', J. Geophys. Res. 70, 5937-5952.

Suess, H.E. (1978) Radiocarbon 20, 1-18.

Tuominen, J., Tuominen, I., and Kyrolinen, J. (1983) 'Eleven-year cycle in solar rotation and meridional motions as derived from the positions of sunspot groups', Mon. Not. Royal Astron. Soc. 205, 691-704.

Weiss, N.O., Cattaneo, F., and Jones, C.A. (1984) 'Periodic and aperiodic dynamo waves', Geophys. Astrophys. Fluid. Dyn. 30, 305-341.

Yoshimura, H. (1975) 'Solar cycle dynamo wave propagation', Astrophys. J. 201, 740-748.

Zeldovich, Ya.B. and Ruzmaikin, A.A. (1983) 'Dynamo problems in astrophysics', Astrophys. and Space Phys. Rev. 2, 333-383.

Zeldovich, Ya.B., Molchanov, S.A., Ruzmaikin, A.A., and Sokoloff, D.D. (1987) 'Intermittency in random medium', Sov. Phys. Uspekhi. 152, 3-32. 